\title{
A IMPORTÂNCIA DA SOCIEDADE BRASILEIRA DE QUÍMICA NA PÓS-GRADUAÇÃo EM QUÍMICA NO BRASIL
}

\author{
Aldo J. G. Zarbin* \\ Departamento de Química, Universidade Federal do Paraná, CP 19032, 81531-980 Curitiba - PR, Brasil
}

Recebido em 09/04/2017; aceito em 23/05/2017

\begin{abstract}
THE IMPORTANCE OF THE BRAZILIAN CHEMICAL SOCIETY FOR THE GRADUATE PROGRAMS IN CHEMISTRY IN BRAZIL. The role of the different actions headed by the Brazilian Chemical Society (SBQ) for the development of the Graduate Programs in Chemistry in Brazil is highlighted herein. Specifically, the importance of the SBQ journals, such as the Quimica Nova (QN), Journal of the Brazilian Chemical Society (JBCS) and Revista Brasileira de Química (RVQ) as vehicles to disseminate the knowledge produced by the different graduate programs in chemistry is demonstrated.
\end{abstract}

Keywords: Brazilian Chemical Society, Graduate Programs in Chemistry, JBCS, Quimica Nova, RVQ.

\section{INTRODUÇÃO}

Poucos meses após sua fundação em julho de 1977, a SBQ lançou a Química Nova, sua primeira e mais longeva revista, que em seu primeiro editorial destacava a importância da ação, e apresentava o periódico como um veículo para a congregação e união da comunidade química brasileira, para divulgação da ciência local na língua local, e para o fortalecimento e estruturação de um "ser coletivo" representativo e respeitado. ${ }^{1}$ Passados 40 anos, o "ser coletivo" se transformou em uma das maiores e mais conceituadas Sociedades Científicas da América Latina. Nesse período a comunidade Química brasileira se fortaleceu e se disseminou por todos os rincões do país, hoje fortemente representada em 70 diferentes Programas de PósGraduação (PPG), responsáveis pela formação de uma média de 1060 mestres e 580 doutores a cada ano, e pela produção de aproximadamente $2 \%$ de todo conhecimento em Química gerado no planeta. E a Química Nova acompanhou de perto toda essa expansão, servindo de estrutura para que fosse possível o nascimento de vários outros membros da família, principalmente os mais próximos Journal of the Brazilian Chemical Society (JBCS), Química Nova na Escola (QNEsc), Boletim Eletrônico da SBQ (BE), e a caçula Revista Virtual de Química (RVQ).

O portfólio de publicações da SBQ vem de encontro às razões de sua existência, como versa o artigo $4^{\circ}$. do seu estatuto, que diz respeito às suas finalidades: "congregar químicos, outros profissionais e instituições ligados à Química, com o propósito de desenvolver, integrar, divulgar e promover a pesquisa, a educação e a aplicação responsável do conhecimento químico, visando ao aumento constante da excelência da Química em todos os aspectos, como forma de impulsionar o desenvolvimento humano e socioeconômico sustentável do País e do mundo". O artigo $5^{\circ}$. lista uma série de 12 diferentes ações que a SBQ poderá realizar visando a consecução dos seus objetivos, onde destaca-se aqui o item I ("promover o prestígio da ciência e de suas aplicações, estimulando qualitativa e quantitativamente a pesquisa e o ensino da Química no País"), e o item II ("manter atividade editorial para divulgar a produção da Química no país e no exterior, por meio de publicações periódicas e monográficas, impressas e/ou eletrônicas, de interesse educacional, científico e tecnológico"), que apresentam um elevado grau de correlação.

\footnotetext{
*e-mail: aldozarbin@ufpr.br
}

As relações diretas entre os diferentes aspectos citados nos parágrafos anteriores são inequívocas, embora muitas vezes não óbvias. A expansão da Química Brasileira está intimamente ligada à expansão da pós-graduação (uma vez que cerca de $90 \%$ de toda a ciência produzida no país é feita pela pós-graduação), e vice-versa. $\mathrm{E}$ a preocupação com todos os aspectos inerentes à pós-graduação (PG) sempre foi (e continua sendo) uma das principais bandeiras da SBQ, em aspectos relacionados a conteúdo, linhas de pesquisa, fomento, bolsas, distribuição regional, critérios de avaliação, políticas de incentivo, suporte para criação de programas, etc. Entretanto, de todas as suas atividades, nenhuma impacta mais a PG em Química no Brasil que a existência das publicações da SBQ, como será apresentado e discutido nesse texto.

\section{A SBQ E A PÓS-GRADUAÇÃO}

A necessidade de se discutir a PG em Química no Brasil de maneira independente foi detectada pela SBQ no início dos anos 2000, quando a PG estava em um processo de expansão. Naquele momento foi criado, pela SBQ, o Fórum dos Coordenadores de PG em Química. O fórum, que foi brilhantemente coordenado dos seus primórdios até 2013 pela Prof. Solange Cadore, do IQ-UNICAMP, e tem hoje por decisão da Diretoria e Conselho (D\&C) o(a) vice-presidente da SBQ como coordenador(a), é um espaço de reflexão permanente e independente, onde os coordenadores de PPGs têm a possibilidade de discutir problemas em conjunto, trocar experiências, e fundamentalmente, propor soluções. A preocupação com os rumos da pesquisa e PG pela SBQ, entretanto, vem dos seus primórdios, e está registrada nas páginas da Química Nova: em 1995, por exemplo, um artigo assinado pela SBQ reproduzia um documento preparado pela D\&C com o objetivo de "refletir sobre a situação da Química no país, bem como contribuir para seu planejamento estratégico nos próximos quatro anos"; ${ }^{2}$ no número especial de comemoração dos 20 anos da SBQ, a evolução da PG foi destacada ${ }^{3}$, além de uma série de análises sobre o papel da SBQ na evolução da Química brasileira, ${ }^{4}$ com um olhar também dedicado às duas décadas da $\mathrm{QN}^{5} \mathrm{e}$ aos 10 anos do JBCS. ${ }^{6}$ Em 2003 os famosos e ainda atuais "Eixos Mobilizadores em Química" apresentavam à comunidade importantes reflexões da SBQ sobre o ensino de graduação e PG em Química, novos modelos de financiamento e perspectivas futuras, através de seis grandes temas e ações. ${ }^{7}$ Mais recentemente, a SBQ capitaneou todo o processo de elaboração de proposta e planejamento do mais novo programa de 
mestrado profissional em Química exclusivamente para professores do ensino médio, o PROFQUI, cujas atividades serão iniciadas no segundo semestre de 2017.

Em que pese a importância de todas as ações discutidas anteriormente, e das inúmeras ações de tão relevante envergadura que não foram aqui citadas, a maior, mais perene e mais importante contribuição da SBQ à PG em Química no Brasil, muitas vezes sem ser devidamente reconhecida, é representada pela qualidade, regularidade e diversidade de suas revistas científicas.

\section{As publicações da SBQ e a Pós-Graduação}

Para os propósitos desse artigo, serão consideradas somente a QN, JBCS e RVQ, por consistirem nos veículos de maior impacto na PG em Química no Brasil, embora seja preciso reconhecer a grande importância da "Química Nova na Escola" nos PPGs em educação. Como mencionado anteriormente, a QN foi a primeira publicação da SBQ, e é uma das mais versáteis e interessantes publicações científicas do país, com artigos contendo resultados originais de pesquisa, revisões, notas técnicas, educação e assuntos gerais em três línguas (português, inglês e espanhol). Além de brasileiros, publicam regularmente em QN autores de vários países, sendo Portugal, Espanha, Irã, Paquistão, Índia, China e os Países da América Latina os mais constantes. O tempo médio entre submissão e aceite em 2016 foi de 2 meses, similar ao tempo entre aceite e publicação na web.

O JBCS teve o seu primeiro número publicado em 1990 e publica artigos em inglês de todos os ramos da química, exceto educação, filosofia e história da química. Com um tempo médio entre a submissão e a publicação na web com o respectivo DOI (Digital Object Identifier) de 3 meses em 2016, o JBCS está cada vez mais atrativo, e tem recebido contribuições de pesquisadores de diferentes países.

A RVQ é uma publicação eletrônica bimestral em língua portuguesa ou inglesa (majoritariamente portuguesa), que visa se constituir de uma fonte de referência e consulta para profissionais e estudantes, de temas referentes a vários domínios da Química. Por ter nascido eletrônica desde a sua concepção, a RVQ tem um caráter mais despojado, e aceita artigos que contenham dados inéditos ou de divulgação, de revisão, sobre atualidades na química brasileira, perfil acadêmico e trajetória científica, métodos de preparação industrial de solventes e reagentes químicos, nomenclatura em química, notícias, debates ou resenhas.

As publicações da SBQ têm todo o seu conteúdo com acesso livre, através dos sítios eletrônicos das revistas ou pelo portal SciELO (Scientific Electronic Library Online) $^{8}$ para QN e JBCS. A manutenção do open access é um princípio fundamental para a SBQ, que se responsabilizava por todos os custos de publicação e editoração até maio/2016, a partir do qual os autores passaram a custear suas publicações, com descontos e subsídios significativos para autores sócios da SBQ.

Aqueles que conhecem e acompanham a trajetória desses três periódicos sabem o alto grau de complementaridade existente entre eles, no tangente aos seus objetivos, públicos, conteúdos, e acessos. Essa complementaridade e diversidade (inclusive de língua de publicação) é um atrativo generoso para que os autores escolham essas revistas como meio de divulgação de seus trabalhos, como se verá a seguir.

Mas como mensurar o impacto dessas três revistas na nossa pós-graduação? Um dos mais importantes parâmetros de avaliação dos PPGs no Brasil foca a qualidade e regularidade da produção científica original decorrente dos trabalhos de teses e dissertações vinculadas. No que diz respeito especificamente à Química, essa produção é fortemente concentrada em periódicos especializados. A classificação dos diferentes periódicos especializados, em cada área do conhecimento, é feita pelo Qualis-Periódicos da CAPES, que corresponde a uma lista organizada de acordo com critérios definidos pela área de conhecimento (e aprovados pelo CTC da CAPES) em estratos em ordem decrescente de importância (A1 o mais elevado, e na sequência, A2, B1, B2, B3, B4, B5 e C, este último com peso nulo), considerando indicativos quantitativos (fatores de impacto) e qualitativos (histórico do periódico, relevância para um determinado segmento, etc.). Há alguns anos o JBCS vem sendo classificado no estrato A2, e a QN e RVQ nos estratos B2 e B3, respectivamente.

De acordo com os dados lançados na Plataforma Sucupira, ${ }^{9}$ todos os PPG em Química no Brasil publicaram em conjunto um total de 10.845 artigos científicos no biênio 2013/2014. A Figura 1(A) representa os 13 periódicos com maior número de publicações pela PG em Química no Brasil nesse biênio, com os respectivos números totais de artigo. É evidente o domínio das publicações da SBQ dentre os veículos de divulgação da PG em Química, com suas três revistas assumindo as três primeiras posições, sendo o JBCS disparado em primeiro lugar com 464 artigos, a QN em segundo com 205 e a RVQ em terceiro com 168. Os três periódicos da SBQ em conjunto divulgaram 7,9\% de toda a produção dos PPG em Química desse biênio, num universo de 1638 diferentes periódicos publicados por editoras de diferentes regiões do planeta. Levando-se em conta somente artigos publicados em revistas classificadas no extrato A, o JBCS também ocupa a primeira posição com larga vantagem, com 453 artigos publicados no biênio, o que representa 13,2\% de todos os trabalhos publicados nesse extrato, e com $335 \%$ mais trabalhos que o segundo colocado. O número total de artigos das 9 revistas mais bem publicadas nesse período no extrato A está ilustrada na Figura 1(B). No que diz respeito às revistas mais procuradas por diferentes PPG em Química (PPGQ), o JBCS também nada de braçadas, como pode ser observado na Figura 1(C). Nesse biênio existiam 61 diferentes PPGQ reconhecidos pela CAPES, e todos publicaram no JBCS. A segunda revista mais procurada foi a RVQ, com artigos publicados por docentes de 39 PPGQs.

A Figura 2 mostra a porcentagem média da produção total dos diferentes PPGQs no ano de 2015 nas revistas da SBQ, de acordo com os conceitos da CAPES. Quando analisados individualmente para cada um dos PPGQs, os dados são extremamente interessantes, e demonstram uma distribuição razoavelmente igualitária (em que pese a existência de raros pontos fora da curva), indicando claramente uma densificação das revistas da SBQ em programas nas diferentes regiões do país e com os diferentes conceitos.

Os dados apresentados anteriormente e sumarizados nas Figuras 1 e 2 são incontestes, mas se tornam ainda mais superlativos quando se levam em conta não só os aspectos quantitativos das publicações da SBQ, mas também o alcance e a visibilidade que estes veículos possuem. Em um trabalho divulgado recentemente no blog "Ciência em Revista", ${ }^{10}$ foi realizado um levantamento sistemático das revistas com maior acesso no portal SciELO no período de setembro/2015 a agosto/2016, separadas nas 8 diferentes áreas de conhecimento reconhecidas pelo CNPq. A QN ocupa um honroso primeiro lugar na área de Ciências Exatas e da Terra, com um total impressionante de 4.197.003 acessos, quase o dobro do segundo colocado que é o JBCS, com expressivos 2.068.866 acessos. Somente para comparação, a terceira colocada é a Acta Amazonica, muito abaixo das duas primeiras, com 879.815 acessos. O Brazilian Journal of Physics ocupa a quinta posição, com 485.088 acessos. Para se ter a exata dimensão desse número, as 8 revistas com maior número de acessos nas Ciências Exatas e da Terra, excetuando-se a QN e o JBCS tiveram, juntas, um total de 3.308.694 acessos, o que corresponde a somente 78,8\% dos acessos da QN. Dentre os 10 artigos mais acessados em cada área do conhecimento, o artigo "Desenvolvimento de métodos de análise por CLAE-UV para os antimicrobianos tetraciclina, sulfametoxazol e trimetoprima utilizando materiais à base de sílica como sistemas 


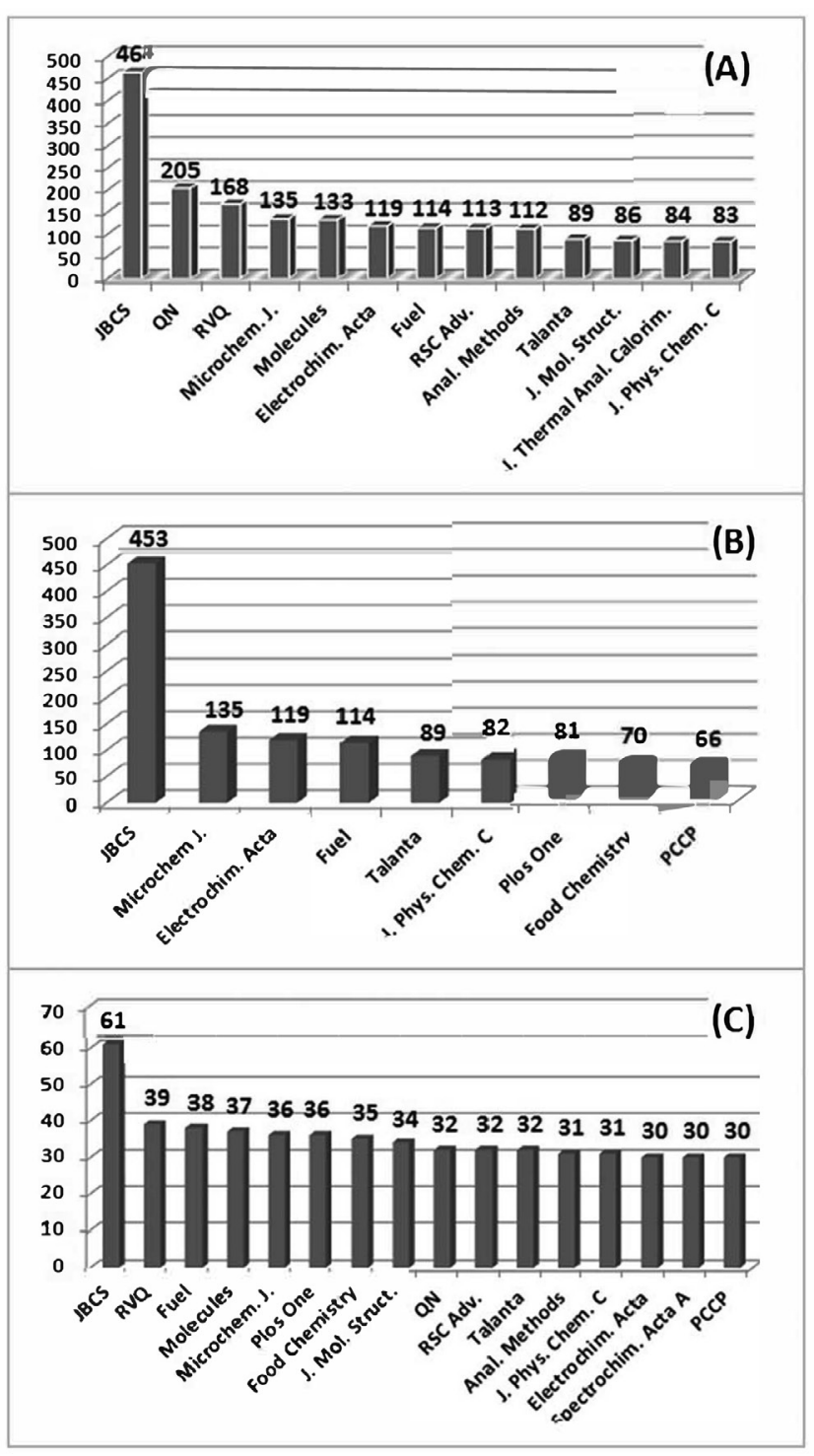

Figura 1. (A) Periódicos com maior número de publicações pela PG em Química no Brasil no biênio 2013/2014, com os respectivos números totais de artigo publicados em cada periódico; (B) número total de artigos publicados pelos $P P G Q$ s nas revistas mais bem publicadas e classificadas no extrato A do Qualis-CAPES, no biênio 2013/2014; (C) número de PPGQ que publicaram nas revistas mais procuradas pelos PPGQ no biênio 2013/2014

de pré-concentração", ${ }^{11}$ publicado em QN, ocupa também o primeiro lugar isolado na grande área das Ciências Exatas e da Terra com 31.426 acessos.

A análise dos números apresentados anteriormente demonstra o tamanho da responsabilidade da SBQ no sistema de Pós-Graduação em Química no Brasil. Mas levanta também algumas reflexões importantes, e a mais evidente delas diz respeito aos Fatores de Impacto (FI) das revistas, em especial da QN e JBCS. O FI da QN em 2016 foi de 0,617 . Levando-se em conta a natureza trilíngue, a predominância dos artigos em português, a possibilidade de publicações de artigos relacionados à discussão de temas relevantes (como os apresentados nesse número especial de comemoração dos 40 anos da SBQ), esse FI é bastante significativo. Por outro lado, o FI não é compatível com o número absurdamente elevado de acessos ao seu conteúdo, como demonstrado anteriormente, numa clara evidência de que os artigos são consultados, mas infelizmente não são citados de forma proporcional. Um dado estarrecedor é que o FI aumentaria

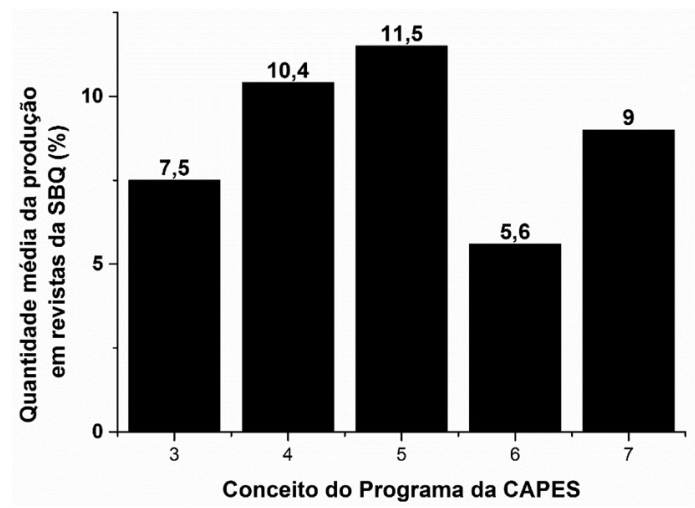

Figura 2. Porcentagem média da produção total dos diferentes $P P G Q s$ no ano de 2015 nas revistas da SBQ, agrupados de acordo com os conceitos da CAPES

significativamente se fossem levados em conta somente os artigos publicados por não-brasileiros na $\mathrm{QN}$, o que significa que estrangeiros citam nossa revista muito mais que os próprios brasileiros.

No tocante ao JBCS, o fator de impacto de 2016 foi de 1,096. Novamente, há duas formas de se interpretar esse dado: se compararmos com periódicos publicados em inglês e editorados por Sociedades Científicas, o FI do JBCS é bastante razoável (o JBCS tem um FI maior que o Canadian Journal of Chemistry, o Journal of the Chinese Chemical Society e o Journal of the Indian Chemical Society, por exemplo). Por outro lado, o FI do JBCS já foi maior (1,539 em 2007), e se considerarmos que os artigos são escritos em inglês, são open access (e portanto acessíveis a toda a comunidade científica global) e que o número de acessos pelo SciELO é extremamente elevado (como mencionado anteriormente), temos novamente uma disparidade entre a realidade e o esperado. Aqui, o diagnóstico é mais sombrio: uma busca não muito elaborada por qualquer pesquisador na base de dados Web of Science, na SciELO ou mesmo no site da PubliSBQ, demonstra que o número de citações de artigos do JBCS nos artigos publicados no próprio JBCS é extremamente baixo (autocitações, menor que $10 \%$ ). Além disso, pesquisadores brasileiros não citam seus próprios trabalhos do JBCS (ou da QN) quando publicam em outras revistas, e fundamentalmente, pesquisadores brasileiros não citam seus colegas brasileiros. Os FIs em potencial da QN e do JBCS seriam facilmente maiores que 1 e 2 , respectivamente, se essas distorções, causadas pela própria comunidade brasileira, fossem corrigidas.

\section{COMENTÁRIOS FINAIS E CONCLUSÕES}

Nos seus 40 anos de história, a SBQ atingiu um patamar de excelência compatível com as grandes sociedades científicas do mundo. Somos uma das maiores do país; temos um padrão de excelência nas nossas revistas e nas nossas reuniões anuais que causam inveja a muitas sociedades coirmãs; temos um reconhecimento internacional que nos faz líderes na América Latina, e parceiros em diversas ações com as maiores Sociedades de Química no planeta, como a americana, a inglesa e a alemã. Esse patamar de excelência foi alcançado pela força e comprometimento com que a SBQ atuou (e atua) nos diferentes segmentos em que interfere, como no sistema nacional de Pesquisa e Pós-Graduação, especificamente demonstrado nesse artigo. Não é nenhum exagero afirmar que todo o sistema de Pós-Graduação em Química do Brasil sofreria um forte abalo sem os alicerces que a SBQ lhe oferece, principalmente através de suas publicações. Infelizmente, o sistema como um todo, principalmente seus maiores beneficiários (orientadores, estudantes de PG e coordenadores dos PPGQs), não dão a tão esperada contrapartida que a SBQ merece, provavelmente por não terem clareza dessa realidade. Existe um PPGQ no Brasil 
que em 2015 teve $18,4 \%$ de toda a sua produção publicada nas revistas da SBQ, mas somente $25 \%$ dos seus docentes permanentes eram sócios da SBQ. Essa discrepância não representa um exemplo isolado, embora não corresponda à maioria dos casos. Muitos atores principais desse processo não fazem correlações causa-efeito, e não entendem que a SBQ existe por e para seus sócios, e deles depende para continuar fazendo sua parte, que como visto aqui, não é pequena.

No discurso de posse como Presidente da SBQ em maio/2016, o autor desse artigo frisou que "a grande comunidade da Química brasileira precisa entender definitivamente que não há uma ciência forte sem uma Sociedade Científica forte por detrás. As centenárias Royal Society of Chemistry e American Chemical Society, com seus mais de 150.000 sócios, são exemplos claros da inequívoca correlação entre a qualidade da Química que se faz em um país e a grandiosidade da sociedade que a representa. Não seremos fortes, enquanto químicos, sem uma SBQ forte e representativa. Para isso é preciso que todos os atores estejam unidos. É preciso que a comunidade química brasileira, na sua totalidade, se veja na SBQ.'

Em novembro de 2016, 45\% dos coordenadores dos Programas de Pós-Graduação em Química do Brasil não eram sócios ativos da SBQ.

\section{AGRADECIMENTOS}

O autor agradece à Prof. E.S. Orth pelo auxílio no levantamento dos dados e discussões; aos Profs. S.I.C. Torresi e W. Loh por alguns dados relativos à QN e JBCS, respectivamente; ao Prof. A. L. Monteiro, pela discussão prévia sobre alguns aspectos da PG em Química no Brasil; à Prof. R.P. Freitas e à funcionária da SBQ R. Fernandes, pelos dados dos sócios ativos da SBQ; aos discentes do GQM-UFPR pelo auxílio na coleta de dados na Plataforma Sucupira.

\section{REFERÊNCIAS}

1. Peixoto, E. M. A.; Quim. Nova 1978, 1, 26.

2. Diretoria e Conselho da SBQ; Quim. Nova 1995, 18, 509.

3. Brocksom, T. J.; de Andrade J. B.; Quim. Nova 1997, 20, 29.

4. Galembeck, F.; Quim. Nova 1997, 20, 5.

5. Ferraz, H. M. C.; Pardini, V. L.; Quim. Nova 1997, 20, 75.

6. Pinto, A. C.; Peixoto, E. M. A; Galembeck F., de Andrade, J. B.; De-Paoli, M. A.; Avaca, L. A.; Alves, O. L.; Quim. Nova 1997, $20,81$.

7. de Andrade, J. B.; Cadore, S.; Vieira P. C.; Zucco, C.; Pinto, A. C.; Quim. Nova 2003, 26, 445.

8. www.scielo.org

9. www.sucupira.capes.gov.br

10. www.blogs.ea2.unicamp.br/cienciaemrevista/2016/10/19/rankingscielo/, acessado em 07/04/2017.

11. Pedroso, R. C. R.; Peralba, M. C. R.; dos Santos, J. H. Z.; Pizzolato, T. M.; Froehlich, P. E.; Quim. Nova 2011, 34, 206. 